\title{
Thermal loading on crystals in an x-ray free-electron laser oscillator
}

\author{
Nanshun Huang $\oplus^{1,2}$ and Haixiao Deng ${ }^{3,1, *}$ \\ ${ }^{1}$ Shanghai Institute of Applied Physics, Chinese Academy of Sciences, Shanghai 201800, China \\ ${ }^{2}$ University of Chinese Academy of Sciences, Beijing 100049, China \\ ${ }^{3}$ Shanghai Advanced Research Institute, Chinese Academy of Sciences, Shanghai 201210, China
}

(Received 15 April 2020; accepted 11 September 2020; published 28 September 2020)

\begin{abstract}
$\mathrm{X}$-ray free electron laser oscillators (XFELOs) are future light sources that produce fully coherent hard $\mathrm{X}$-ray pulses. Based on the low-gain principle, the XFELO traps $\mathrm{X}$-ray pulses in an optical cavity composed of multiple Bragg-reflecting mirrors that have high reflectivity in a bandwidth of about ten meV. The crystal mirrors exposed to intense $\mathrm{x}$-ray beams in the optical cavity are subject to thermal deformations that would shift and distort the Bragg reflectivity curve. Therefore, the stability of the XFELO operation depends on the ability of the mirrors to maintain the Bragg reflection under such thermal load. A new approach was used to analyze the thermal load of the mirrors. The approach utilizes a dedicated Bragg reflection physical process in GEANT4 to obtain precise absorption information of the XFELO pulses in the crystal. Following transient thermal behavior, including single pulse and multiple pulse inputs, was analyzed by finite element analysis software based on the energy absorption information extracted from the GEANT4 simulation. It is shown that, for a typical XFELO pulse depositing about ten microjoules energy the over a spot of tens of micrometers in radius, the thermal relaxation time across the thickness is on tens of nanoseconds scale. In this situation, a simplified heat-load model is then developed to integrate the heat load in the XFELO. With the simplified model, the potential impact of the thermal load on the XFELO operation is estimated. When a large amount of heat remains in the crystal, the pulse energy drops significantly and has large oscillations due to negative feedback of the temperature change on the pulse energy.
\end{abstract}

DOI: 10.1103/PhysRevAccelBeams.23.090704

\section{INTRODUCTION}

In the hard $\mathrm{x}$-ray regime, the operating free electron lasers (FELs) are based on the SASE (self-amplified spontaneous emission) mode [1-5], which can generate $x$-ray pulses with unique characteristics, such as ultrahigh peak power and ultrashort pulse duration. However, originating from shot noise, the stochastic nature of SASE leads to a low temporal coherence and poor pulse-to-pulse stability of produced $\mathrm{x}$-ray pulses. To obtain stable, fully coherent $\mathrm{x}$-ray pulses with statistical properties similar to a fully coherent optical laser, an x-ray free electron laser oscillator (XFELO) has been proposed [6-8]. In an XFELO, the X-ray pulses amplified by the interaction with electron bunches in a short undulator, circulate in a low-loss optical cavity formed by multiple Bragg-reflecting crystals, which have a reflectivity close to $100 \%$ within a bandwidth of $\sim 10 \mathrm{meV}[9,10]$ in the hard x-ray regime, whereas conventional mirrors are unavailable.

\footnotetext{
*denghaixiao@zjlab.org.cn
}

Published by the American Physical Society under the terms of the Creative Commons Attribution 4.0 International license. Further distribution of this work must maintain attribution to the author(s) and the published article's title, journal citation, and DOI.
Generally, the operation of XFELOs is based on an accelerator that can deliver electron bunches with a repetition rate of $1 \mathrm{MHz}$ level or above. With high-brightness electron bunches at $\mathrm{MHz}$ repetition rate, the intracavity $\mathrm{x}$-ray pulses could have a pulses energy of about $800 \mu \mathrm{J}$ and beam radius of about $30 \mu \mathrm{m}$. Such intense x-ray pulses inevitably impose a high heat load on the mirrors, which result in lattice distortions in the crystal. Since the Bragg reflection originates from the $\mathrm{x}$-ray scattering of a periodic atomic lattice, the lattice distortions would decrease the peak of Bragg reflectivity, shift reflectivity curves over the spectrum, and increase sidebands $[11,12]$. Therefore, the realization of the optical cavity with Bragg-reflecting mirrors relies highly on the stability of the crystal lattice. The heat load of the crystal mirrors under such intense $\mathrm{x}$-ray pulses is an essential issue of building an XFELO.

In order to investigate the influence of the heat load in crystals, two kinds of experiments have been performed [13-19]. The first one utilizes a high power conventional laser in the long-wavelength regime to model laser-mirror interactions. The advantage of this method is the ability of precisely controlling the pulse duration and pulse energy, which is crucial for light-material interactions. However, this approach produces a penetration depth at nanometer level [14], which is far shorter than that produced by $\mathrm{x}$-rays. Besides, the repetition rate of the high power conventional 
laser is challenging to reach $\mathrm{MHz}$ or higher. Another method applies the synchrotron radiation light source. The synchrotron radiation light source can generate hard $\mathrm{x}$-rays with sufficient time-averaged power. However, the synchrotron radiation is polychromatic, and the monochromator is required to obtain high spectral purity. With the monochromator, the pulse energy of the synchrotron radiation light sources decreases significantly [15]. Besides, if an x-ray beam generated by synchrotron radiation is micro-focused to the XFEL beam level [18], its angular spreads may be much larger than Bragg reflection acceptance. Thus, in general, the experimental conditions do not meet the requirements of XFELO operation, and it is still necessary to numerically model the heat load of mirrors and its feedback on XFELO operation.

In previous studies [20-22], the numerical evaluation of the $\mathrm{x}$-ray absorption in the crystal uses an exponential attenuation model that describes the intensity of the x-rays decreases to $1 / \mathrm{e}$ of initial value through one attenuation length. Although the exponential attenuation model is feasible in most of the x-ray interactions [23,24], it needs to be improved in the $\mathrm{x}$-ray Bragg reflection. Therefore, this paper proposes a simple new physical process to describe the Bragg reflection in GEANT4, a powerful particle tracker. Combined with the other implemented physical process in the GEANT4, a complete set of interactions can be provided in the simulation of light-material interactions. Based on the heat source obtained by GEANT4 simulations, transient thermal analysis can be conducted with finite-element analysis (FEA) software, ANSYS [25]. Furthermore, by coupling the temperature evolution of the crystals into the XFELO simulations, one can gain the ability to investigate the impact of the thermal loading on an XFELO operation.

This paper is organized as follows. The Sec. II presents the characteristics of Bragg reflections relating to the thermal load. In the Sec. III, the light-matter interaction is simulated in GEANT4, and the following thermal behavior is analyzed. In the Sec. IV, a simplified model to integrate the thermal loading into XFELO simulations is presented. Then, the simulations of thermal loading coupled XFELO are performed. A summary is presented in the Sec. V.

\section{CHARACTERISTICS OF BRAGG REFLECTIONS}

In an XFELO, the single-pass gain is $G$, while the total reflectance is $R$. When $(1+G) R>1$, the x-ray pulses could evolve from the initial noise to intense coherent radiation. The oscillator reaches steady state when $(1+G) R=1$. As the single-pass gain $G$ is governed by the electron beam qualities and undulator configurations, the ability to achieve the stable XFELO operation relies on the reflectivity $R$, which is sensitive to the lattice structure of the crystals. Adverse effects including the thermal deformations and mounting vibrations of the crystals, may emerge to affect the

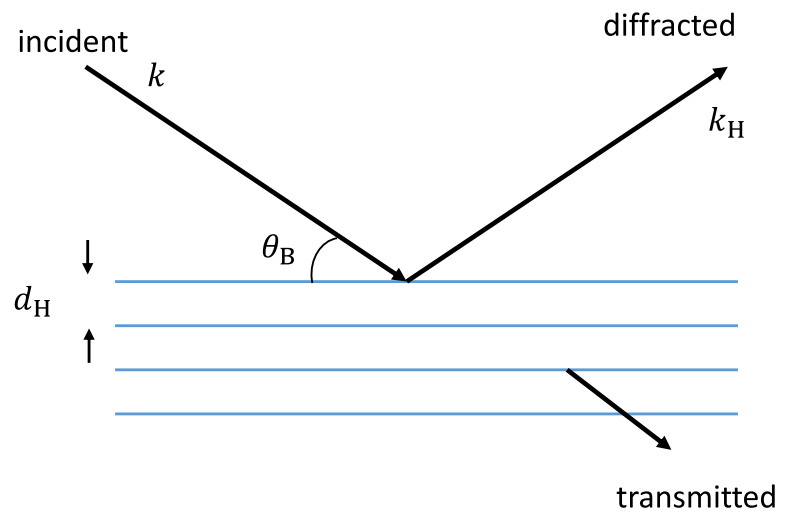

FIG. 1. A symmetric x-ray diffraction. The blue lines are the virtual atomic planes. $k$ is the wave number of incident $\mathrm{X}$-ray, and $k_{\mathrm{H}}$ is reflected one. $\theta_{\mathrm{B}}$ is the Bragg angle.

lattice and to disrupt the Bragg reflection. Here, we present a brief introduction of the relationship between the lattice distortion and the Bragg reflection.

The Bragg reflection or diffraction originates from the $\mathrm{x}$-ray scattering of atoms in a periodic structure [26], which can be imagined as a reflection of imaginary "mirrors" formed by atomic planes in the crystal lattice, seen in Fig. 1. The Bragg condition can be expressed as

$$
n \lambda_{\mathrm{B}}=2 d_{\mathrm{H}}(T) \sin \theta_{\mathrm{B}},
$$

where $n$ is the diffraction order, $\theta_{B}$ is the angle of incidence, $\lambda_{B}$ is the radiation wavelength at which the reflection occurs, $d_{H}$ is the distance between the atomic planes and $T$ is the temperature. The lattice planes is described by the Miller indices, $h, k, l$, three integers. The spacing between the lattice planes is given by $d_{\mathrm{H}}(T)=\frac{a(T)}{\sqrt{h^{2}+k^{2}+l^{2}}}$, where $a$ is the lattice constant. Diamond is a preferred material that can be employed by a high power XFELO to form the X-ray cavity, as it is a unique combination of outstanding thermal and optical properties, including a high thermal diffusivity, low thermal expansion, and high Bragg reflectivity for $\mathrm{x}$-rays [27]. In the following discussions, the crystal mirror is assumed to be the diamond.

As expected, the high reflectivity in Bragg reflection is only possible if the lattice structure is perfect for a large amount of diffracting planes. In general, the change of crystal lattice acts on both the position and the amplitude of the Bragg reflectivity $[11,12]$. Figure 2 shows three kinds of lattice distortions (up panel) and corresponding effect (bottom panel). In each condition, the undistorted lattice (black dot) is compared with the strained lattice (red dot). The lower plots present the influence of these distortions on the reflectivity curve: (a) Random disorder decreases the peak reflectivity of the Bragg reflection; (b) Homogeneous linear strains shift the reflection; (c) Longitudinal nonlinear strains create sidebands and decrease the peak value of the Bragg reflectivity. 

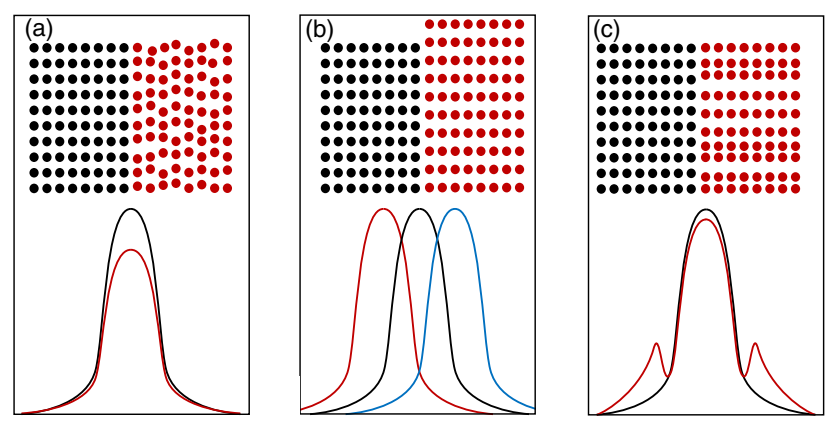

FIG. 2. Three schematic presentations of lattice distortion. In each case, the strained crystal (red dot) is compared with undistorted lattice (black dot). The lower panel shows the impact of the distortion on the reflectivity curve of Bragg reflection. (a) Random disorder decreases the Bragg peaks. (b) Homogeneous deformations lead to spectral shifts depending on the relative change of the lattice parameter. (c) Longitudinal nonlinear strains create sidebands and decrease the Bragg peaks.

When a high-brilliance $\mathrm{x}$-ray pulse is reflected by a Bragg-reflecting crystal, a certain part of the energy is deposited. Different positions and deposited energies would imprint different distortions into the crystal. The type (a) distortion occurs in a case of very high energy density that may impose radiation damage to the crystal. In this case, the crystal may totally lose the long-range order with a set of irreversible changes. In the case of far below the damage threshold, the absorbed energies due to the light-matter interactions translate into the other disorders, type (b) and (c), which are the major issues in the XFELO. When the considered temperature map is complex, the corresponding reflectivity curves can be obtained by solving the multidimensional Takagi-Taupin equations with the strain profile [28]. If the temperature change is homogeneous, one mainly considers the variation of the lattice constant.

In principle, the relative change of the lattice constant can be described by the thermal expansion coefficient, $\beta$. For a cubic crystal like the diamond, the relative change owing to the thermal expansion can be expressed as:

$$
\delta_{a}=\exp \left(\int_{T_{0}}^{T} \beta\left(T^{\prime}\right) T^{\prime} d\right)-1
$$

While the temperature change is small, $\beta$ can be assumed constant and relative change of lattice constant can be approximated to $\frac{a(T)-a\left(T_{0}\right)}{a\left(T_{0}\right)} \approx \beta \times\left(T-T_{0}\right)$ with the first order Taylor series. The thermal expansion coefficient $\beta(T)$ can be obtained by an empirical formula from Ref. [27], which can be written as $\beta=4.25 \times 10^{-14} T^{3}$.

As Eq. (2) has shown, a small expansion coefficient is expected to increase the tolerance of the heat load on the crystal. Therefore, it is preferable to use the crystals mirrors at a low temperature, such as $100 \mathrm{~K}$, where the thermal expansion coefficient drops by a few orders of magnitude in comparison with the room temperature value of about $1 \times 10^{-6}$. Additionally, the thermal conductivity of diamond would reach the maximum value in the temperature range from $60 \mathrm{~K}$ to $100 \mathrm{~K}$ [29].

\section{LIGHT-MATTER INTERACTIONS AND HEAT CONDUCTIONS}

In order to investigate the lattice distortions of mirrors, simulating abilities are necessary for obtaining accurate and reliable information of the absorbed energies in the crystals. This raises an essential problem with the interactions between the XFELO radiation pulses and the crystal mirrors. In this section, with the help of a dedicated Bragg reflection model, the light-matter interactions have been numerically simulated within GEANT4 toolkit [30], which is developed and actively used for particle experiments and detector designs. GEANT4 is a Monte Carlo based toolkit for simulating the particle interacting with matter. It includes many functionalities like tracking, geometry, physics models, and hits. For convenience and practicality, it already implements many widely used physics models to describe the fundamental light-matter interactions.

Based on the low energy Livermore model in GEANT4 [31], an additional Bragg reflection process has been implemented to extend the available range of particle interactions. The implemented Bragg reflection model could redirect photons in the crystal under the Bragg condition. Thus, this model naturally enjoys the feature of the multiple reflecting, which is the most important consideration for Bragg reflection and is absent from the previous exponential attenuation model. As the Bragg reflection occurs in a range of ten $\mathrm{meV}$ level, the table that stores the cross section of each interaction in the GEANT4 kernel must be extended to reach the meV level. The cross section of the Bragg reflection, the probabilities considered in the GEANT4 simulation, is calculated via the dynamical theory of $\mathrm{x}$-ray diffraction. The cross section is given by

$$
\sigma_{\mathrm{B}}(\mathrm{b} / \text { atom })=\mu / \rho\left(\mathrm{cm}^{2} \mathrm{~g}^{-1}\right)\left\{m_{\mathrm{u}}(\mathrm{g}) A\right\} \times 10^{24},
$$

where $1 / \mu$ is the photon-energy dependent excitation length calculated by the dynamical theory $[9,32], m_{\mathrm{u}}=$ $1.66053886 \times 10^{-24} \mathrm{~g}$ is the atomic mass unit, and $A$ is the relative atomic mass of the target element. In addition, a parameter defined as the probability that a reflected $\mathrm{x}$-ray will be reflected again can be used to control the multiple Bragg reflections and to correct the reflections in thicker crystals. Overall, in the view of implemented and defined components, all aspects of the Bragg reflection simulation have been involved: (a) the geometry of the diamond crystal; (b) the primary particle (photons); (c) the generation of particles; (d) the tracking of particles through materials; (e) the physics processes modeling particle 


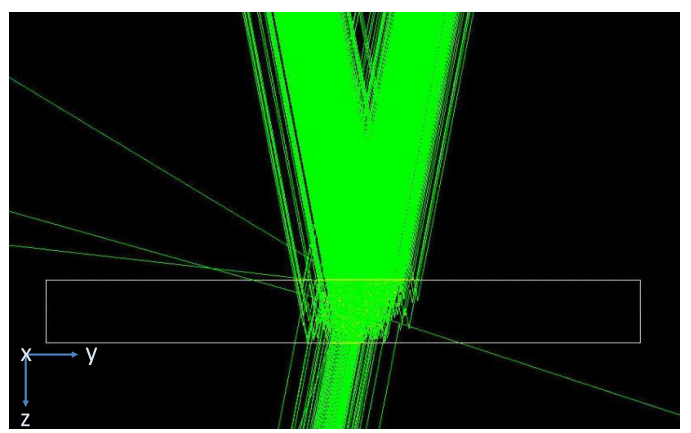

FIG. 3. A snapshot of the light-matter interactions in the GEANT4 simulation. The green line is the x-rays. The white box is the frame of the crystal. A large part of incident $\mathrm{x}$-rays is reflected, with crystal thickness of $70 \mu \mathrm{m}$, photon energy of $14.33 \mathrm{keV}$ and Bragg angle of about $83^{\circ}$. The incident direction of the $\mathrm{x}$-rays points to the negative value of the $\mathrm{z}$-axis.

interactions (photoelectric effect, Compton scattering, pair production, bremsstrahlung, and Bragg reflection.).

Figure 3 shows a snapshot of the particle tracking in the crystals related to (3 37 ) diffracting planes, while the photon energy is about $14.33 \mathrm{keV}$ with an FWHM bandwidth of $3 \mathrm{meV}$. As it is shown, the Bragg reflections govern the interaction between the X-rays with the crystal. The absorptions of the x-rays mainly result from the photoelectric effect and Compton scattering. According to the statistical data from the GEANT4 simulation, the diamond mirror with a thickness of $70 \mu \mathrm{m}$ allows reflecting $87 \%$ of the incident $\mathrm{x}$-ray while the transported part remains $11.7 \%$. In other words, only $1.3 \%$ of the incident $x$-ray has been absorbed. Since the dynamical theory of $\mathrm{x}$-ray diffraction predicts the peak reflectance to be $88 \%$ and transmittance to be $11.7 \%$, the GEANT4 simulation results are reasonable and acceptable.

Another benchmark about the evolution of the transverse intensity profile is shown in Fig. 4. The GEANT4 results are compared to those of solving two-dimensional TakagiTaupin equations. The reflected intensity profiles of the two cases have a slight difference, while the incident pulses have the same profile. These results show that the dedicated model including multiple reflections in GEANT4 can simulate the x-ray crystal interactions with reasonable accuracy.

To obtain the absorption data used for thermal analysis, the GEANT4 simulation utilizes a set of baseline parameters based on the previous studies of XFELO operation for SHINE (Shanghai high repetition rate XFEL and extreme light facility), the first hard $\mathrm{x}$-ray free electron laser in China [33-35]. The cavity is formed by four diamond mirrors with $\left(\begin{array}{lll}3 & 3 & 7\end{array}\right)$ diffracting planes while the cavity configuration is optimized to amplify the $\mathrm{x}$-ray pulse with a photon energy of $14.33 \mathrm{keV}$. The intracavity pulse energy is assumed to be $600 \mu \mathrm{J}$ with a $1 \mathrm{MHz}$ repetition rate and 3 meV FWHM spectral width. The temporal distribution of $\mathrm{x}$-ray photons is assumed to a Gaussian distribution with an RMS length of $12 \mu \mathrm{m}$. Meanwhile, the pulse has a

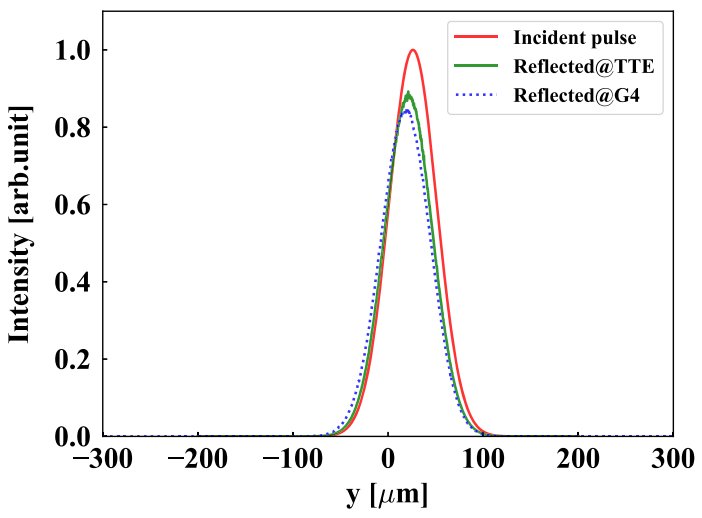

FIG. 4. A benchmark of the GEANT4 (G4) reflection process. The G4 results are compared to those of solving two-dimensional Takagi-Taupin equations (TTE). The reflected intensity profiles of the two cases have a reasonable difference, while the incident pulses have the same profile. The G4 simulation and the TTE calculation use an RMS transverse size of $25 \mu \mathrm{m}$.

Gaussian transverse profile with an RMS size of $25 \mu \mathrm{m}$, while the divergence angle is assumed to be $0.7 \mu \mathrm{rad}$, which is much smaller than Bragg reflection widths. In addition, the crystal size is $1000 \mu \mathrm{m} \times 1000 \mu \mathrm{m} \times 70 \mu \mathrm{m}$ in the GEANT4 simulation.

Considering the pulse energy of $600 \mu \mathrm{J}$, the total absorbed energy is about $7.3 \mu \mathrm{J}$ in the diamond crystal during the reflection. The transverse distribution of the absorbed energy is mainly determined by the transverse profile of the incident $\mathrm{x}$-ray pulse, seen in Fig. 5. With an RMS spot size of about $25 \mu \mathrm{m}$, the maximum energy density reaches $2400 \mu \mathrm{J} / \mathrm{mm}^{2}$ corresponding to timeaveraged power of $2.4 \mathrm{~kW} / \mathrm{mm}^{2}$ at $1 \mathrm{MHz}$ repetition rate.

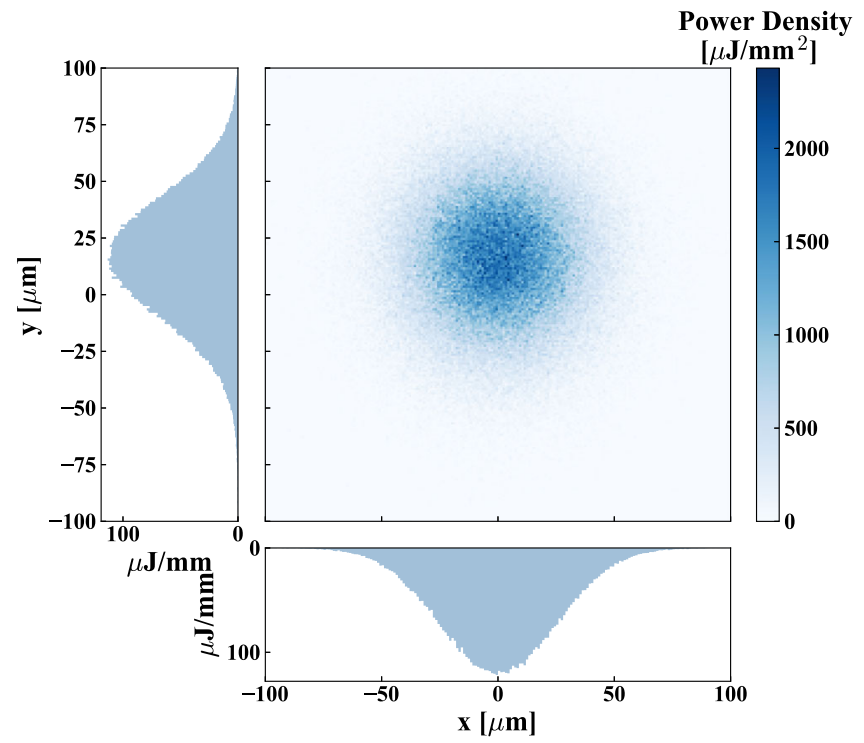

FIG. 5. The density of absorbed energy over the transverse plane. The corresponding marginal distribution is also shown. The maximum energy density reaches $2400 \mu \mathrm{J} / \mathrm{mm}^{2}$. 
Since the crystal rotates about $7^{\circ}$ along the $\mathrm{x}$-direction to fulfill the Bragg condition, the irradiation spot size in Fig. 5 on y-direction is a little larger than that of $\mathrm{x}$-direction. This is due to the transverse shift as X-ray penetrates the crystal at a non-normal angle of incidence. When the Bragg angle is small, this effect would significantly affect the transverse distribution of absorbed energy. It is difficult to evaluate the multiple reflections in theoretical formulas, but simple in this GEANT4 model.

The distributions of the absorbed energy on $\mathrm{x}-\mathrm{z}$ plane (top) and y-z plane (bottom) are shown in Fig. 6. The tilted penetration is presented in the bottom plot of Fig. 6. Due to the short excitation length associated with a high reflectance, the incident x-ray pulse is significantly attenuated (reflected). The exponential decay model predicts an almost uniform trend when the thickness is only $70 \mu \mathrm{m}$. In contrast, this model shows that the absorption is concentrated in the part of the crystal near the incident plane due to the high reflectivity. For thinner crystals, there are few multiple reflections of x-rays in the crystal, and the absorption is very similar to that predicted by the exponential decay model. Since this model can give more details about the absorbed energy, it could be a powerful tool to study the thermal load of XFELO.

Another advantage is that this scheme allows the study of dynamic absorption, the absorption energy at different times, seen in Fig. 7. In this case, the time at zero is the
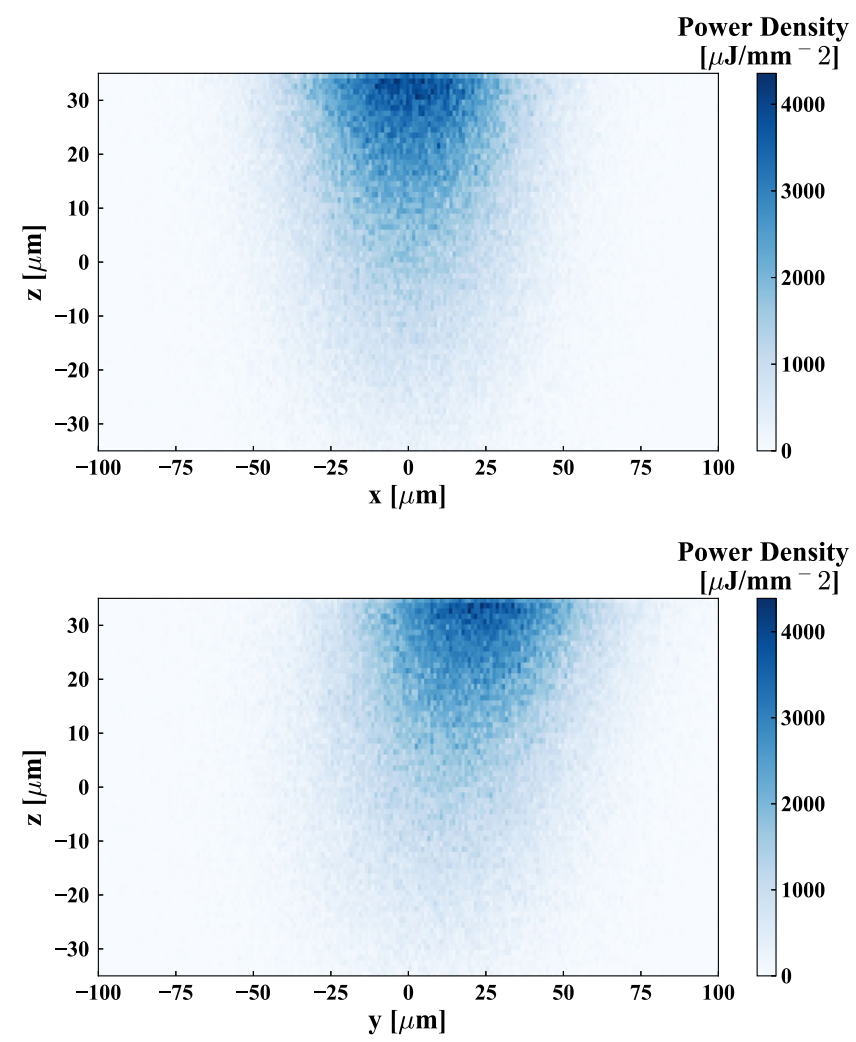

FIG. 6. The distribution of absorbed energy in $x-z$ plane (top) and $\mathrm{y}-\mathrm{z}$ plane (bottom).

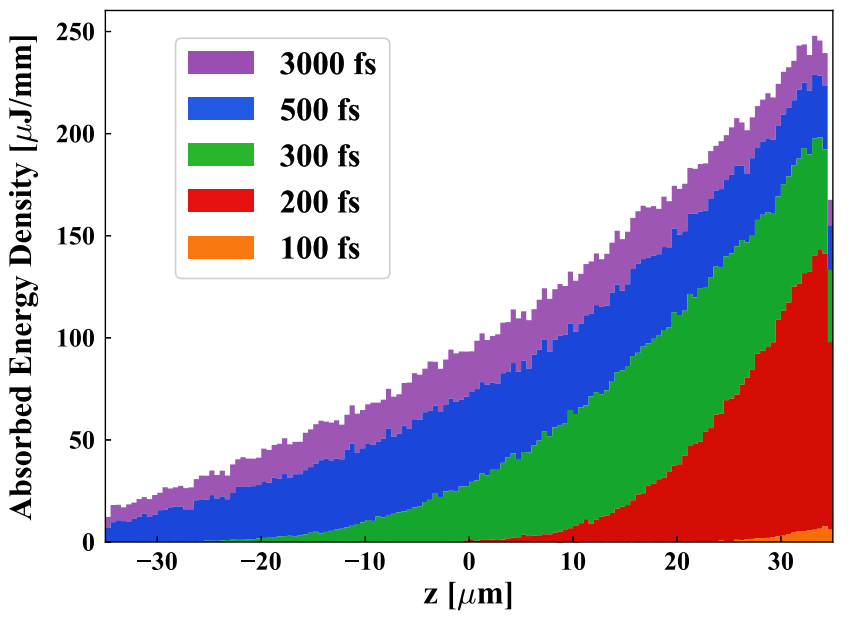

FIG. 7. Absorbed energy density along the thickness at different times.

time when the first photon enters the crystal. At $500 \mathrm{fs}$, most of the interaction between the x-ray and the crystal has been completed. The duration of the light-matter interaction is determined by the temporal duration of the x-ray pulse and the thickness of the crystal. In addition, with the help of molecular dynamics simulation software, it is possible to study the possible dynamic changes of the crystal during the pulse duration. But, this is not our intent here.

Since the XFELO pulses may be too short on the timescale to affect their own Bragg reflection [36,37], one can assume that the crystal temperature variation only acts on the subsequent pulses. Therefore, it can be assumed that the $\mathrm{x}$-ray pulses induce instantaneous heating in the crystal. In other words, the energy deposition resulting from the light-matter interactions is converted into a transient temperature increment.

To study the thermal behavior of the diamond crystal, the commercial finite element analysis software ANSYS was used [25]. The initial temperatures were obtained from the absorption information from the GEANT4 simulation. In the ANSYS transient thermal analysis, the crystal size is $800 \mu \mathrm{m} \times 800 \mu \mathrm{m} \times 70 \mu \mathrm{m}$, which is large enough to cover the irradiation. The incident and transmitted surface are insulated, while the other surrounding surfaces are fixed at $70 \mathrm{~K}$. The work in Ref. [29,38,39] is adopted to obtain the thermal conductivity and specific heat capability. As it is various as a function of the temperature, a fitting linear polynomial formula is utilized for simplicity. The mesh size is optimized, and the time step is fixed to be $0.5 \mathrm{~ns}$.

The thermal conduction calculations for the single pulse input are shown in Figs. 8 and 9. Figure 8 shows temperature snapshots at 1, 3, 5, 10, 250, and $1000 \mathrm{~ns}$ after irradiation. Figure 9 shows the evolution of temperature changes $(T-70 \mathrm{~K})$ at the center of the incident $(z=35 \mu \mathrm{m})$ surfaces and transmitted $(z=-35 \mu \mathrm{m})$ surfaces, while the temperature change averaged over the entire crystal volume is presented by a green dash line. 

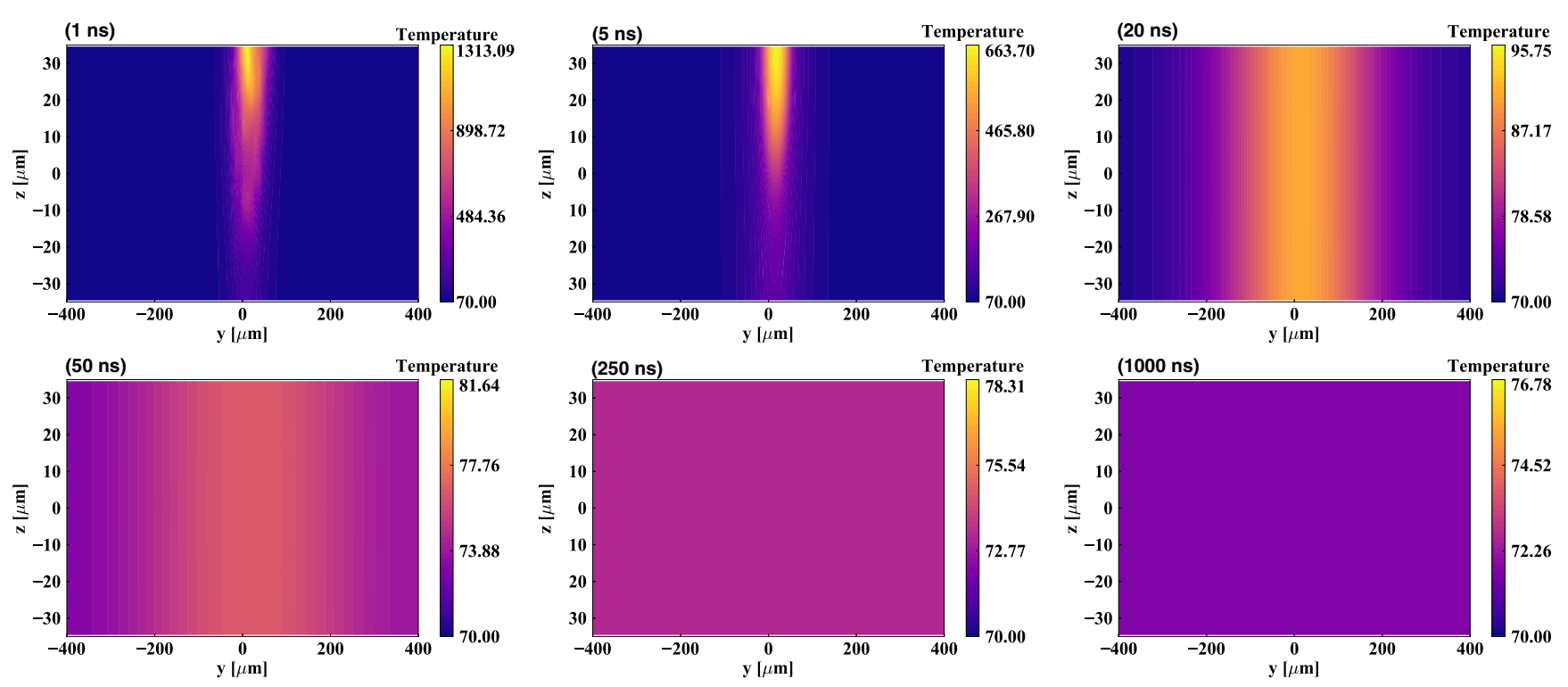

FIG. 8. Snapshots of temperature field calculated with a single-pulse input. It can be seen that the early temperature is much higher on the incident surface, and the highest temperature drops rapidly to $95 \mathrm{~K}$ within $20 \mathrm{~ns}$. At $20 \mathrm{~ns}$, it can be found that the temperature along the thickness becomes homogeneous. The temperature is nearly uniform throughout the entire crystal at $250 \mathrm{~ns}$ and drops to about $71.8 \mathrm{~K}$ at $1000 \mathrm{~ns}$.

As shown in Fig. 8, the initial temperature of the incident surface is higher than that of the backside due to the Bragg reflection. The highest temperature near the center of the incident surface is about $1300 \mathrm{~K}$. It decreases rapidly in the first $50 \mathrm{~ns}$ and gradually slows down in the following $100 \mathrm{~ns}$. This is due to that the thermal conductivity increases rapidly during the first $50 \mathrm{~ns}$ thermal diffusion, and then the thermal diffusion slows down as the temperature gradient gradually decreases. As suggested by the almost identical temperatures of the incident surface and the backside in Fig. 8 and Fig. 9, the thermal relaxation time across the whole thickness is considered to be about

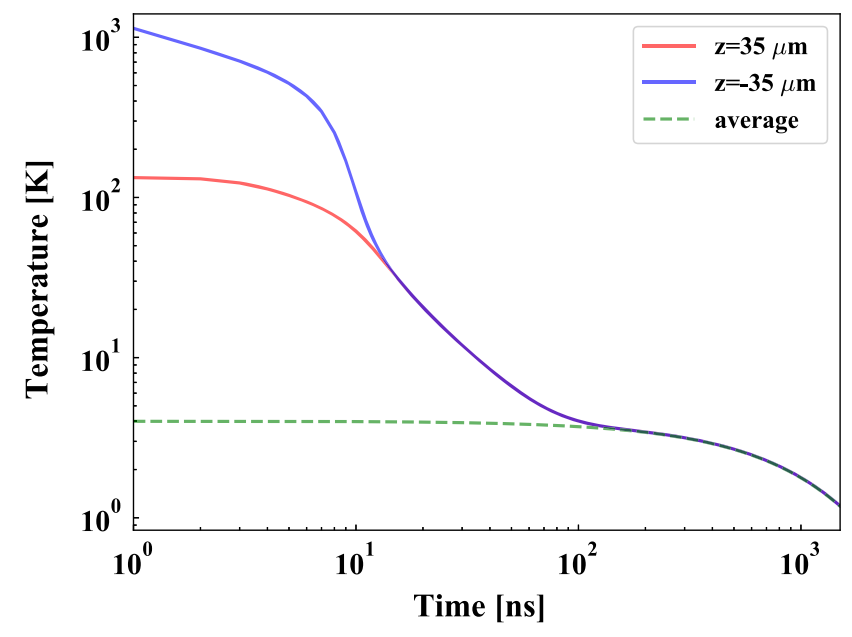

FIG. 9. The temperature (minus $70 \mathrm{~K}$ ) at the center of the incident surface $(z=35 \mu \mathrm{m})$ and transmitted surface $(z=-35 \mu \mathrm{m})$ is shown. The temperature averaged over the whole crystal is presented by a green dash line.
20 ns. Meanwhile, thermal relaxation time in the radial direction exceeds $150 \mathrm{~ns}$. Indeed, the temperature is nearly homogeneous on the whole crystal after 200 ns. The maximum temperature difference of the whole crystal is about $0.1 \mathrm{~K}$ at $250 \mathrm{~ns}$ and decreases to $0.05 \mathrm{~K}$ at $500 \mathrm{~ns}$. The mean temperature increase is about $4 \mathrm{~K}$, with a total absorbed energy of $7.3 \mu \mathrm{J}$. In the following $1000 \mathrm{~ns}$, the mean temperature change decreases to about $1.8 \mathrm{~K}$, which corresponds to a relative change in lattice parameters of $1 \times 10^{-8}$. At $2000 \mathrm{~ns}$, the mean temperature change drops to $0.6 \mathrm{~K}$. It still has a downward trend, but will decrease over a longer period of time.

The multipulse results are shown in Fig. 10. The power of each laser pulse is added periodically. The time step is fixed at $1 \mathrm{~ns}$, while the other parameters are the same as above. Simulations were performed with $20 \mu$ s, 20 pulses at $1 \mathrm{MHz}$. The temperature rises instantaneously after each pulse. Its evolution in the interpulse period was similar to the single-pulse case described above. However, the average temperature keeps increasing because some residual heat remains in the crystal. Thus, the temperature increment consists of two components: (1) the heating due to x-ray irradiation and (2) the long-term accumulation of residual heat in the crystal. In our case, the temperature increment due to the irradiation is about $4 \mathrm{~K}$, while the accumulated temperature increment is about $3.5 \mathrm{~K}$. The accumulation of heat places a practical requirement on the cooling system to ensure that the accumulated temperature is trivial. The solution should be established in the specific context of XFELO operation, and is not our intent here. The specific cooling system design can learn experiences from the monochromator design on the synchrotron light source. 


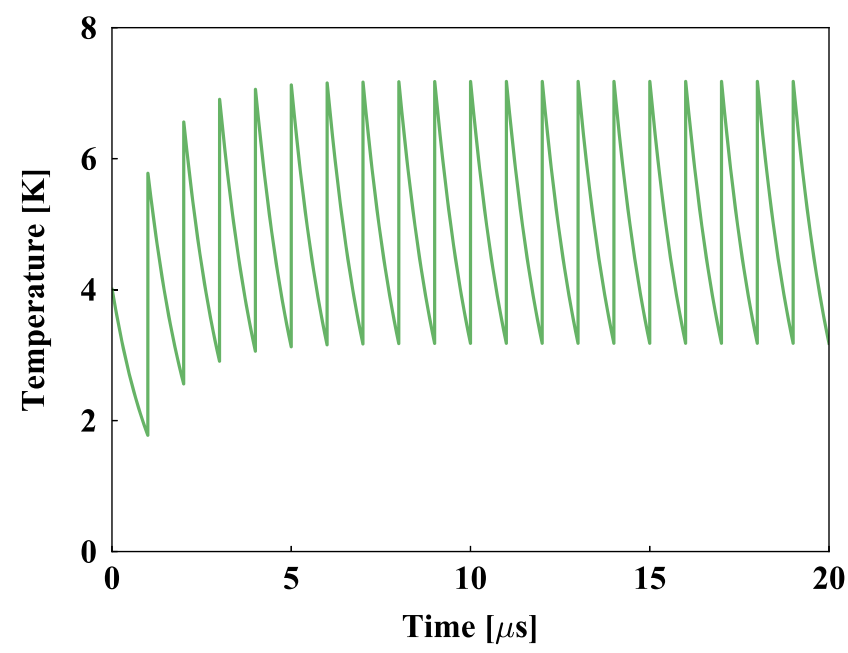

FIG. 10. Time histories of the averaged temperature (minus $70 \mathrm{~K}$ ). The repetition rate is $1 \mathrm{MHz}$, and the periodic input laser pulse is the same as the single-pulse one.

\section{THERMAL LOADING COUPLED XFELO SIMULATION}

The thermal load affects the Bragg reflection mainly through the deformation of the lattice. As mentioned in Sec. II, the pulse energy determines that the type (a) distortion is negligible in XFELO. Furthermore, according to the transient thermal simulation above, the type (c) distortion that requires a temperature gradient across the thickness is also negligible. This is because of that the thermal relaxation time across the thickness at low temperatures is very short compared to the interpulse time. Thus, when considering the long-term accumulation, the temperature gradient over the entire thickness is negligible. Therefore, it is reasonable to assume that the most critical issue with the XFELO thermal load is the shift of the reflectivity curve due to the uniform expansion of the crystal.

Therefore, the simplified model could focus on the temperature change averaged over the effective volume, which is $8 \sigma_{r} \times 8 \sigma_{r} \times d$ where $\sigma_{r}$ is the RMS photon beam size, $d$ is crystal thickness. This assumption is based on the fact that the thermal conductivity of the diamond is very high and that the beam size is much smaller than the crystal size. Then, a simplified model of the averaged temperature can be expressed as [40]:

$$
\frac{d \Delta T}{d t}=-\alpha \times \Delta T
$$

where $\Delta T=T-T_{0}$ is the temperature change averaged over the effective volume, $\alpha$ is the temperature spreading rate associated with thermal conductivity, specific heat, and crystal size. When the temperature does not change significantly, the value of $\alpha$ can be assumed to be constant. Then, for the single-pulse input, the solution can be written as

$$
\Delta T=\Delta T_{p} e^{-\alpha t},
$$

where $\Delta T_{p}$ is the single-pulse heating temperature. With a stable repetition rate, the time for cooling is constant. Thus, we can define $\eta=e^{-\alpha \Delta t}$ as the cooling efficiency, with repetition rate of $1 / \Delta t . \eta$ describes residual temperature after a period of $\Delta t$. Its approximation value can be obtained from the transient thermal analysis of single-pulse input. In the multiple-pulse case, the averaged temperature change can be written as:

$$
\Delta T_{n+1}=\left(\Delta T_{n}+\Delta T_{p}\right) \eta(\eta<1),
$$

where $n$ indicates nth pulse. At steady state, the averaged temperature change become stable and we can get a solution, $\Delta T_{n+1}=\Delta T_{n}=\eta \Delta T_{p} /(1-\eta)$. Then, the shift of reflectivity can be written as $\Delta E \approx E_{H} \beta \Delta T_{n}$, where $E_{H}$ is the Bragg energy.

With the help of the simplified thermal loading model, a thermal loading coupled XFELO is performed based on the parameters of SHINE. The main parameters of SHINE are listed in Table I. SHINE equips a superconducting linac that can deliver electron bunches with energy of $8 \mathrm{GeV}$ and $100 \mathrm{pC}$ total charge compressed to a peak current of $700 \mathrm{~A}$.

The optical cavity is built from four diamond $(3,3,7)$ crystal mirrors, seen in Fig. 11. With a thickness of $70 \mu \mathrm{m}$, the downstream diamond mirror $C_{4}$ at $14.33 \mathrm{keV}$ has $87 \%$ reflectivity (coupling output is 12\%). The other three mirrors are expected to reach the total reflection with thick crystals. Since the three crystals do not need to consider the transmission of the output coupling, the temperature is appropriate to be fixed by $70 \mathrm{~K}$. In the simulation, the heat load effect is mainly considered in the downstream mirror $\mathrm{C}_{4}$ for simplicity.

The simulations are conducted by using the combination of a time-dependent FEL code GENESIS [41], a field propagation simulation code OPC [42], and a Bragg reflection simulation code BRIGHT [43]. The simplified thermal loading

TABLE I. The main parameters of XFELO operation for SHINE.

\begin{tabular}{lc}
\hline \hline Parameter & Value \\
\hline Beam energy & $8 \mathrm{GeV}$ \\
Relative energy spread & $0.01 \%$ \\
Normalized emittance & $0.4 \mathrm{~mm} \cdot \mathrm{mrad}$ \\
Peak current & $700 \mathrm{~A}$ \\
Charge & $100 \mathrm{pC}$ \\
Undulator period length & $16 \mathrm{~mm}$ \\
Undulator segment length & $4 \mathrm{~m}$ \\
Photon energy & $14.33 \mathrm{keV}$ \\
Mirror material & Diamond \\
Peak reflectivity & $87 \%$ \\
Darwin width & $11 \mathrm{meV}$ \\
Coupling-out mirror thickness & $70 \mu \mathrm{m}$ \\
\hline \hline
\end{tabular}




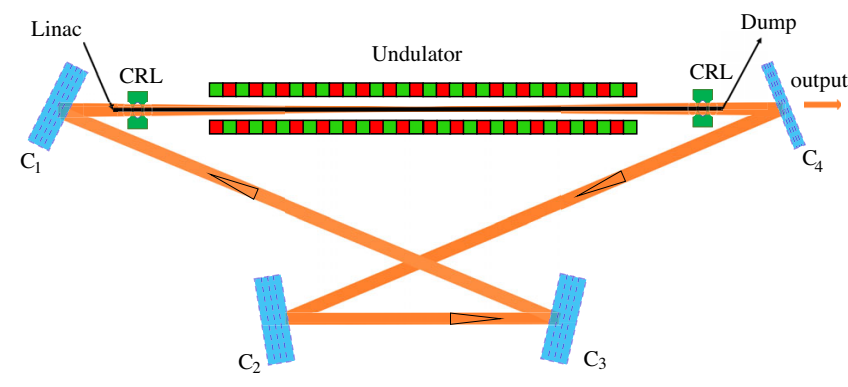

FIG. 11. A schematic XFELO configuration for the SHINE. Four diamond mirrors are used. The photon energy is $14.33 \mathrm{keV}$. $C_{1}, C_{2}$, and $C_{3}$ are thick crystal to reach a total reflection. The x-ray coupled out from the downstream mirror $C_{4}$ which peak reflectivity reaches $87 \%$.

model is integrated into the BRIGHT as the part of the Bragg reflection. In the following analysis, the x-ray pulse is indicated as the intra-cavity one to present how much energy is imposed to the mirrors. The output value needs to be multiplied by an output-coupling factor, the transmittance.

The simulations were conducted while $\eta$ is $0.1,0.5,0.75$, and 0.95 . The spectrum and power profile are presented in Fig. 12 and Fig. 13, respectively. When $\eta=0.95$, a shift in the reflectivity of $C_{4}$ due to the heat load can be found in the top plot of Fig. 12. Additionally, the Bragg reflectivity of $C_{1}$ is shown by an orange dash line. As expected, in the spectrum, the FEL pulse is trapped in the overlap between the two reflectivity curves. Therefore, the total integral reflectance changes dramatically when the shift in the position of the Bragg reflectivity exceeds half of the Darwin width. This effect significantly influences the stability of the optical cavity and the gain process of XFELOs. Thus, the peak power decreases significantly as $\eta$ rises, as shown in the bottom plot of Fig. 12. Another fact should be noted is that the different reflectivities also yields a slight difference in time delay, which results from the penetration during the Bragg reflection. The mismatch of time delay leads to the imperfect temporal overlap between the $\mathrm{x}$-ray pulses and the electron beam, which can decrease the peak power further. It may be a crucial issue for short electron bunches.

Figure 13 shows the detailed simulation results, including the spectrum evolution and pulse energy growth when $\eta$ is $0.1,0.5,0.75$, and 0.95 . The left panel of Fig. 13 presents the energy evolutions (red line) and the corresponding temperature change (green line). With a small $\eta(0.1)$, the temperature change is trivial, and the system is stable. The pulse energy reaches about $1 \mathrm{~mJ}$ at saturation, while the corresponding temperature change is only $0.8 \mathrm{~K}$. At the steady state, pulse energy has a very small oscillation, which is an XFELO nature. When $\eta$ is set to 0.5 , the temperature change increase to $10 \mathrm{~K}$, while the saturated pulse energy decrease to about $900 \mu \mathrm{J}$. The decrement of the pulse energy is mainly due to the decrement of total integral reflectivity.
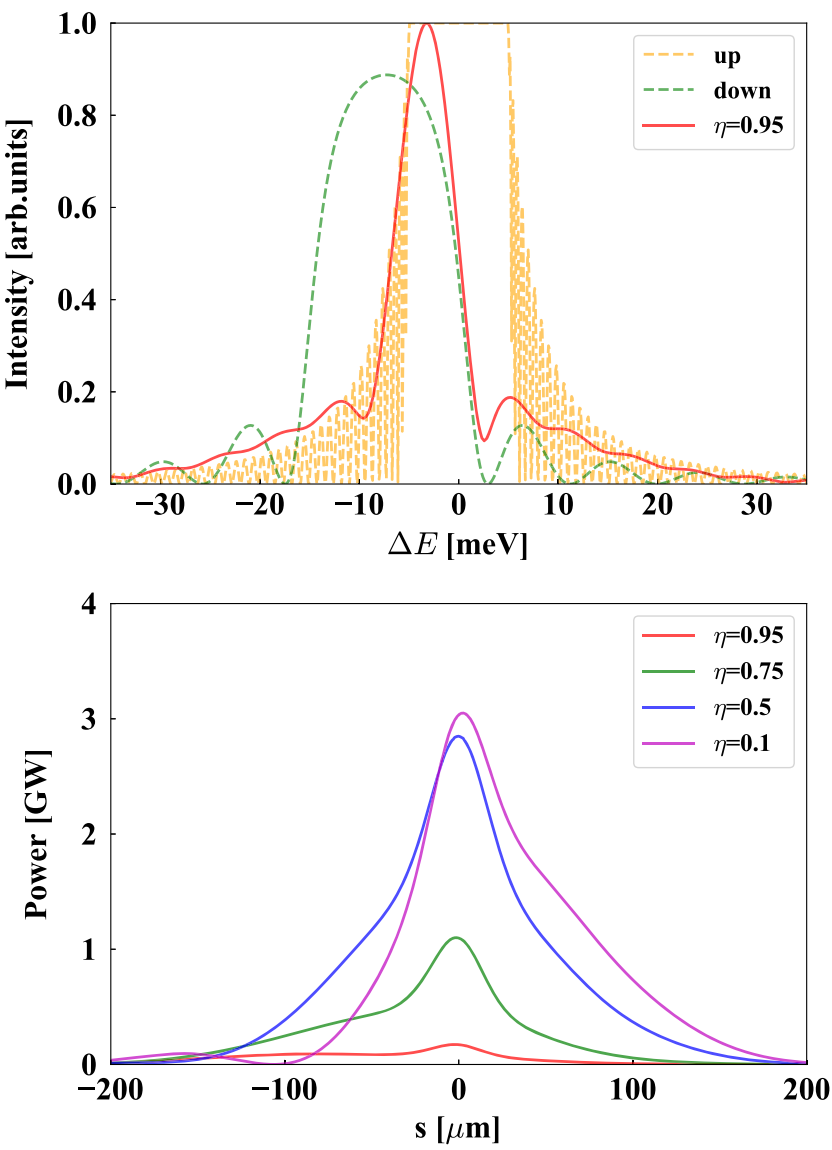

FIG. 12. The spectrum of XFELO pulse is shown in the top plot where $\eta$ is 0.95 . The Bragg reflectivity as a function of photon energy for downstream mirror $C_{4}$ (green) and upstream mirror $C_{1}$ (orange) is presented by dash lines. Shifts in the spectrum and reflectivity can be found. The power profile for each $\eta$ is shown in the bottom plot. The maximum peak power exceeds $3 \mathrm{GW}$.

When a large amount of heat remains in the crystal, the pulse energy has large oscillations. The source of the oscillations is the negative feedback of the temperature change on the pulse energy. Since negative feedback generally promotes stability, the XFELO may reach a steady state with the negative feedback of temperature change, such as $\eta$ is 0.75 . However, the steady-state pulse energy is about $350 \mu \mathrm{J}$, only a third of the value of $\eta=0.1$. In addition, the temperature change always lags behind the pulse energy variation. The combined result is that the temperature peak gradually approaches the trough of the pulse energy in time. Thus, when $\eta=0.95$ corresponding to a poor cooling capability, the system cannot reach steady state.

The evolution of the spectrums is presented in the right panel of Fig. 13. The simulations share the same initial random number. Thus, each $\eta$ has a similar period for building up the longitudinal coherence of the x-ray pulse. As energy increases, the spectral width decreases further. The final FWHM spectral width is nearly $13 \mathrm{meV}$ when $\eta$ is 0.1 or 0.5 . The value decreases to about 9 mev at $\eta=0.75$ due to the narrower overlap in reflectivity. The maximum 

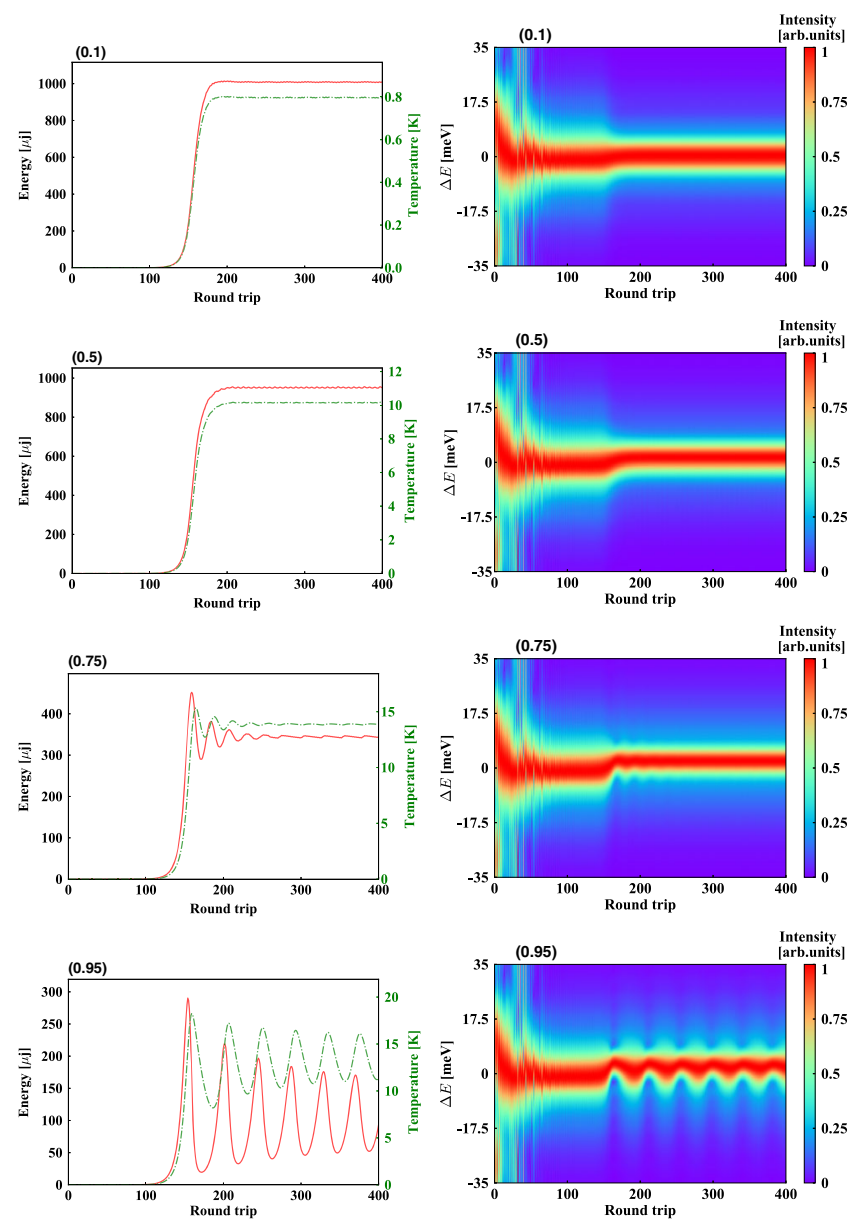

FIG. 13. The evolution of the pulse energy (red line) and the averaged temperature (minus $70 \mathrm{~K}$, green line) at various $\eta$ : 0.1 , $0.5,0.75$, and 0.95 . The evolution of the spectrum at various $\eta$ : $0.1,0.5,0.75$, and 0.95 . In each round trip, the spectrum is normalized by its maximum value.

available shift is about $6 \mathrm{meV}$, which is nearly half of the Darwin width. When the reflectivity shift exceeds this value, the spectrum oscillates along with the pulse energy.

\section{CONCLUSION}

In this paper, we have performed a numerical model to study the heat load of the mirrors in the XFELO operation, including the fundamental light-material interactions, the thermal behavior analysis, and the corresponding heat-load coupled XFELO simulations. The light-material interactions were simulated by means of the particle tracking package GEANT4 with the dedicated Bragg reflection physical process. This dedicated model can give accurate information of $\mathrm{x}$-ray absorptions in the Bragg-reflecting crystals. Following transient thermal behavior, including single pulse and multiple pulse inputs, was analyzed by finite element analysis software based on the energy absorption information extracted from the GEANT4 simulation. It is shown that, for a typical XFELO pulse depositing about ten microjoules energy the over a spot of tens of micrometers in radius, the thermal relaxation time across the thickness is on tens of nanoseconds scale. In this situation, a simplified heat-load model is then developed to integrate the heat load in the XFELO. With the simplified model, the potential impact of the thermal load on the XFELO operation is estimated. With a large residual fraction of the temperature, the pulse energy and peak power were found to decrease markedly due to the reflectivity shifting and time delay mismatching. The simulations also indicate that the pulse energy and spectrum may have large oscillations due to negative feedback of the temperature change on the pulse energy.

In addition to this work, the analysis of non-Fourier heat conductions as well as the thermal vibrations will be performed to better understand the various thermal load effects. Furthermore, the mirror cooling system for the XFELO operation of SHINE will be designed and optimized in the future.

\section{ACKNOWLEDGMENTS}

The authors are grateful to J. Yan and K. Li for helpful discussions and useful comments. This work was partially supported by the National Key Research and Development Program of China (Grants No. 2018YFE0103100, No. 2016YFA0401900) and the National Natural Science Foundation of China (Grants No. 11935020, No. 11775293).

[1] W. Ackermann et al., Operation of a free-electron laser from the extreme ultraviolet to the water window, Nat. Photonics 1, 336 (2007).

[2] P. Emma et al., First lasing and operation of an ångstromwavelength free-electron laser, Nat. Photonics 4, 641 (2010).

[3] T. Ishikawa, H. Aoyagi, T. Asaka, Y. Asano, N. Azumi, T. Bizen, H. Ego, K. Fukami, T. Fukui, Y. Furukawa et al., A compact $\mathrm{X}$-ray free-electron laser emitting in the sub-ångström region, Nat. Photonics 6, 540 (2012).

[4] H.S. Kang et al., Hard X-ray free-electron laser with femtosecond-scale timing jitter, Nat. Photonics 11, 708 (2017).

[5] C. Milne, T. Schietinger, M. Aiba, A. Alarcon, J. Alex, A. Anghel, V. Arsov, C. Beard, P. Beaud, S. Bettoni et al., SwissFEL: The Swiss X-ray free electron laser, Appl. Sci. 7, 720 (2017).

[6] K. J. Kim, Y. Shvyd'ko, and S. Reiche, A Proposal for an X-Ray Free-Electron Laser Oscillator with an EnergyRecovery Linac, Phys. Rev. Lett. 100, 244802 (2008).

[7] R. R. Lindberg, K. J. Kim, Y. Shvyd'Ko, and W. M. Fawley, Performance of the X-ray free-electron laser oscillator with crystal cavity, Phys. Rev. Accel. Beams 14, 010701 (2011).

[8] J. Dai, H. Deng, and Z. Dai, Proposal for an X-Ray Free Electron Laser Oscillator with Intermediate Energy Electron Beam, Phys. Rev. Lett. 108, 034802 (2012). 
[9] A. Authier, Dynamical Theory of X-Ray Diffraction (Oxford University Press, New York, 2010), https://doi.org/ 10.1093/acprof:oso/9780198528920.001.0001.

[10] Y. Shvyd'ko, S. Stoupin, V. Blank, and S. Terentyev, Near$100 \%$ Bragg reflectivity of X-rays, Nat. Photonics 5, 539 (2011).

[11] A. Rousse, C. Rischel, and J. C. Gauthier, Femtosecond x-ray crystallography, Rev. Mod. Phys. 73, 17 (2001).

[12] M. Bargheer, N. Zhavoronkov, M. Woerner, and T. Elsaesser, Recent progress in ultrafast X-ray diffraction, Chem. Phys. Chem. 7, 783 (2006).

[13] S. Kojima, K. Y. Llu, Y. Kudo, S. Kawado, T. Ishikawa, and T. Matsushita, Time-resolved X-ray diffraction measurement of silicon surface during laser irradiation under grazing-incidence conditions, Jpn. J. Appl. Phys. 33, 561 (1994).

[14] B. Perrin, Investigation of Short-Time Heat Transfer Effects by an Optical Pump-Probe Method, in Top. Appl. Phys., Topics in Applied Physics, edited by S. Volz (Springer Berlin Heidelberg, 2006), Vol. 107, pp. 333359, https://doi.org/10.1007/11767862_13.

[15] P. Van Aerenbergh, C. Detlefs, J. Härtwig, T. A. Lafford, F. Masiello, T. Roth, W. Schmid, P. Wattecamps, and L. Zhang, High heat load diamond monochromator project at ESRF, AIP Conf Proc. 1234, 229 (2010).

[16] S. Stoupin, S. A. Terentyev, V. D. Blank, Y. V. Shvyd'Ko, K. Goetze, L. Assoufid, S. N. Polyakov, M. S. Kuznetsov, N. V. Kornilov, J. Katsoudas, R. Alonso-Mori, M. Chollet, Y. Feng, J. M. Glownia, H. Lemke, A. Robert, M. Sikorski, S. Song, and D. Zhu, All-diamond optical assemblies for a beam-multiplexing X-ray monochromator at the Linac Coherent Light Source, J. Appl. Crystallogr. 47, 1329 (2014).

[17] M. Kozák, F. Trojánek, and P. Malý, Hot-carrier transport in diamond controlled by femtosecond laser pulses, New J. Phys. 17, 053027 (2015).

[18] T. Kolodziej, Y. Shvyd'ko, D. Shu, S. Kearney, S. Stoupin, W. Liu, T. Gog, D. A. Walko, J. Wang, A. Said, T. Roberts, K. Goetze, M. Baldini, W. Yang, T. Fister, V. Blank, S. Terentyev, and K. J. Kim, High Bragg reflectivity of diamond crystals exposed to multi-kW mm${ }^{-2}$ X-ray beams, J. Synchrotron Radiat. 25, 1022 (2018).

[19] L. Samoylova, D. Shu, X. Dong, G. Geloni, S. Karabekyan, S. Terentev, V. Blank, S. Liu, T. Wohlenberg, W. Decking, and H. Sinn, Design of hard x-ray self-seeding monochromator for European XFEL, AIP Conf. Proc. 2054, 030016 (2019), https://aip.scitation.org/doi/pdf/10.1063/1.5084579.

[20] M. Q. Song, Q. M. Zhang, Y. H. Guo, K. Li, and H. X. Deng, Numerical modeling of thermal loading of diamond crystal in X-ray FEL oscillators, Chin. Phys. C 40, 048101 (2016).

[21] B. Yang and S. Wang, and J. Wu, Transient thermal stress wave and vibrational analyses of a thin diamond crystal for $\mathrm{X}$-ray free-electron lasers under high-repetition-rate operation, J. Synchrotron Radiat. 25, 166 (2018).

[22] S. Liu, W. Decking, V. Kocharyan, E. Saldin, S. Serkez, R. Shayduk, H. Sinn, and G. Geloni, Preparing for highrepetition rate hard x-ray self-seeding at the European X-ray Free Electron Laser: Challenges and opportunities, Phys. Rev. Accel. Beams 22, 060704 (2019).
[23] S. Seltzer, X-Ray Mass Attenuation Coefficients (1996), https://doi.org/10.18434/T4D01F.

[24] B. L. Henke, E. M. Gullikson, and J.C. Davis, X-ray interactions: Photoabsorption, scattering, transmission, and reflection at $E=50-30,000 \mathrm{eV}, Z=1-92$, At. Data Nucl. Data Tables 54, 181 (1993).

[25] H.-H. Lee, Finite Element Simulations with ANSYS Workbench 17 (SDC Publications, 6800 Squibb Road Mission, KS 66202, 2017).

[26] J.H. Hubbell and I. Overbo, Relativistic atomic form factors and photon coherent scattering cross sections, J. Phys. Chem. Ref. Data 8, 69 (1979).

[27] S. Stoupin and Y. V. Shvyd'Ko, Ultraprecise studies of the thermal expansion coefficient of diamond using backscattering x-ray diffraction, Phys. Rev. B - Condens. Matter Mater. Phys. 83, 1 (2011).

[28] A. P. Honkanen, C. Ferrero, J. P. Guigay, and V. Mocella, A finite-element approach to dynamical diffraction problems in reflection geometry, J. Appl. Crystallogr. 51, 514 (2018).

[29] A. V. Inyushkin, A. N. Taldenkov, V. G. Ralchenko, A. P. Bolshakov, A. V. Koliadin, and A. N. Katrusha, Thermal conductivity of high purity synthetic single crystal diamonds, Phys. Rev. B 97, 144305 (2018).

[30] J. Allison et al., Recent developments in Geant4, Nucl. Instrum. Methods Phys. Res., Sect. A 835, 186 (2016).

[31] G. A. Cirrone, G. Cuttone, F. Di Rosa, L. Pandola, F. Romano, and Q. Zhang, Validation of the Geant4 electromagnetic photon cross-sections for elements and compounds, Nucl. Instrum. Methods Phys. Res., Sect. A 618, 315 (2010).

[32] Y. Shvyd'Ko and R. Lindberg, Spatiotemporal response of crystals in x-ray Bragg diffraction, Phys. Rev. Accel. Beams 15, 100702 (2012).

[33] Z. Zhao, D. Wang, Z.-H. Yang, and L. Yin, SCLF: An 8-GeV CW SCRF Linac-Based X-Ray FEL Facility in Shanghai, in Proceedings, 38th International Free Electron Laser Conference, FEL2017 (2018), p. MOP055, https://doi.org/10.18429/JACoW-FEL2017-MOP055.

[34] K. Li and H. Deng, Gain-guided X-ray free-electron laser oscillator, Appl. Phys. Lett. 113, 061106 (2018).

[35] J. Yan and H. Deng, Multi-beam-energy operation for the continuous-wave x-ray free electron laser, Phys. Rev. Accel. Beams 22, 090701 (2019).

[36] N. Medvedev, V. Tkachenko, V. Lipp, Z. Li, and B. Ziaja, Various damage mechanisms in carbon and silicon materials under femtosecond X-ray irradiation, 4Open 1, 3 (2018).

[37] K. Sokolowski-Tinten and D. Von Der Linde, Ultrafast phase transitions and lattice dynamics probed using laserproduced x-ray pulses, J. Phys. Condens. Matter 16, R1517 (2004).

[38] L. Wei, P. K. Kuo, R. L. Thomas, T. R. Anthony, and W. F. Banholzer, Thermal Conductivity of Isotopically Modified Single Crystal Diamond, Phys. Rev. Lett. 70, 3764 (1993).

[39] R. R. Reeber and K. Wang, Thermal expansion, molar volume and specific heat of diamond from 0 to 3000k, J. Electron. Mater. 25, 63 (1996).

[40] B. F. Shorr, Foundations of Engineering Mechanics Thermal Integrity in Mechanics and Engineering (Springer, New York, 2015), https://doi.org/10.1007/9783-662-46968-2. 
[41] S. Reiche, GENESIS 1.3: a fully 3D time-dependent FEL simulation code, Nucl. Instrum. Methods Phys. Res., Sect. A 429, 243 (1999).

[42] J. G. Karssenberg, P. J. Van Der Slot, I. V. Volokhine, J. W. Verschuur, and K. J. Boller, Modeling paraxial wave propagation in free-electron laser oscillators, J. Appl. Phys. 100, 093106 (2006).

[43] N. S. Huang, K. Li, and H. X. Deng, BRIGHT: The threedimensional X-ray crystal Bragg diffraction code, Nucl. Sci. Tech. 30, 39 (2019). 